\title{
Comparação entre diferentes métodos de interpolação zonal para estimativa populacional: estudo de caso das áreas urbanas do Distrito Federal
}

Comparison between different methods of zonal interpolation for population estimate: Case study of the Federal District urban areas

\author{
Kássia Batista de Castro ${ }^{1}$ \\ Henrique Llacer Roig ${ }^{2}$ \\ Marina Rolim Bilich Neumann ${ }^{3}$
}

Recebido em agosto de 2018. Aprovado em dezembro de 2018.

\begin{abstract}
RESUMO
Os dados acerca das características da população, normalmente divulgados pelo censo, são de suma importância, pois subsidiam inúmeros estudos sociais, econômicos e ambientais. Estes dados são agregados de maneira arbitrária, considerando a informação homogênea em um determinado espaço. Uma alternativa para contornar esse problema e gerar informação com qualidade espacial mais precisa é a utilização do mapeamento dasimétrico. $\mathrm{O}$ mapa dasimétrico utiliza dados auxiliares para refinar a representação da distribuição espacial da variável analisada. No decorrer das últimas décadas essa se tornou uma técnica bastante utilizada e diversos trabalhos foram realizados com diferentes abordagens metodológicas. Dessa forma, este trabalho tem como objetivo avaliar o desempenho de três diferentes abordagens do mapeamento dasimétrico para a estimativa da população urbana do Distrito Federal. Para tanto, utilizou-se os modelos dasimétricos por ponderação de área (MD1), utilizando densidade relativa subjetiva (MD2) e o método dasimétrico inteligente (MDI) com a densidade relativa amostrada pelo método do centroide (MD3). Para a geração dos mapas foram utilizados dados do censo com o valor populacional em nível de subdistrito. O mapa de uso e cobertura utilizado possui resolução de $1 \mathrm{~m}$ e foi modificado a partir do mapeamento de tipos de estruturas urbanas (UST) do DF, o qual classifica a área urbana em relação a sua homogeneidade considerando aspectos de funcionalidade,
\end{abstract}

\footnotetext{
1 Programa de Pós-graduação em Geociências Aplicadas, Universidade de Brasília, Brasil. E-mail: kassiabcastro@gmail.com

2 Instituto de Geociências, Universidade de Brasília, Brasil. E-mail: roig@unb.br

3 Faculdade de Agronomia e Medicina Veterinária, Universidade de Brasília, Brasil. E-mail: marinabilich@unb.br
} 
material de cobertura e características físicas. Para a avaliação dos métodos gerados utilizou-se métodos estatísticos e gráficos, utilizando como comparação a informação da população em nível de setores censitários. De acordo com as análises aplicadas o modelo que demonstrou o melhor desempenho foi o MD3, sendo esta técnica aplicada para o nível de informação de setores censitários como mapa final de estimativa da distribuição espacial da população urbana do DF.

PALAVRAS-CHAVE: Mapeamento dasimétrico, crescimento urbano, UST.

\begin{abstract}
The data about population characteristics, normally released by the census, are of paramount importance because subsidize numerous social, economic and environmental studies. These data are aggregated arbitrarily considering the homogeny information in a given space. An alternative to work around this problem and generate information with more accurate spatial quality is the use of dasymetric mapping. The dasymetric mapping uses auxiliary data to refine the representation of the spatial distribution of the variable analyzed. During the last decades it has become a widely used technique, and many studies have been conducted with different methodological approaches. Thus, this study aims to evaluate the performance of three different approaches of dasymetric mapping to estimate the urban population of the Federal District. For this purpose, we used the dasymetric model per areal weighting (MD1);using subjective relative density (MD2) and; intelligent dasymetric method (IDM) with relative density sampled by the centroid method (MD3). For the generation of maps census data were used with the population value in the sub-district level. The land cover map used has a resolution of $1 \mathrm{~m}$ and was modified of the mapping of urban structures types (UST) of the Federal District, which ranks the urban area in relation to its homogeneity considering aspects of functionality, covering material and physical characteristics. For the evaluation of the generated methods used statistical methods and graphs, using as comparison information of the population at the level of census tracts. According to the analysis applied the model demonstrated the best performance was the MD3, with this technique applied to the census tracts information level as final statement of estimated of the spatial distribution of the urban population of the Federal District.
\end{abstract}

KEYWORDS: Dasymetric mapping, urban growth, UST.

$$
* * *
$$

\title{
Introdução
}

A população urbana mundial teve um rápido crescimento passando de 746 milhões em 1950 para 3,9 mil milhões em 2014, hoje, 55\% da população mundial vive em áreas urbanas, uma proporção que se espera venha a 
aumentar para 66\% em 2050. As projeções mostram que a urbanização associada ao crescimento da população mundial poderá trazer mais 2,5 mil milhões de pessoas para as populações urbanizadas em 2050 (ONU, 2014; ONU, 2016). E de acordo com a última projeção acerca do crescimento populacional mundial (ONU, 2015), a população atual de 7,3 mil milhões deve alcançar os 8,5 mil milhões em 2030, 9,7 mil milhões em 2050 e 11,2 mil milhões em 2100.

O aumento da população apresenta reflexos nos mais diversos setores, desde impactos econômicos e sociais, como saúde, desigualdade e desemprego (NERY et al., 2007; RÉQUIA JR. et al 2015; CRUZ et al., 2016), como para o meio ambiente, seja decorrente da pressão por alimentos e matéria-prima (CARDOSO et al., 2010; GODFRAY et al., 2010; WIEDMANN et al., 2015) ou da ocupação do espaço pelo homem (MENNIS 2002; SLEETER e WOOD, 2006; BRANDÃO et al., 2015). Como acontece com qualquer variável geográfica, a forma como os dados demográficos são recolhidos e disponibilizados tem implicações para a sua manipulação e representação, bem como a natureza complexa dos dados da população coloca vários desafios para a modelagem e análise de sua distribuição no espaço e no tempo de forma adequada (FREIRE, 2007).

$\mathrm{Na}$ maioria dos países, assim como no Brasil, os dados acerca das características populacionais são estimados a cada dez anos pelo censo (FRANÇA et al., 2014). Os dados censitários são agregados em unidades de zoneamento, cujos limites são arbitrários (administrativos ou para facilitar a coleta), para preservar a individualidade e a identidade das informações (LANGFORD e UNWIN, 1994; FRANÇA et al., 2014).

A informação pode ser agregada em diferentes níveis (estadual, municipal, em subdistritos ou outros) de acordo com o que se almeja analisar. De uma maneira geral, o menor nível de agregação é o setor censitário. Um setor censitário pode conter muitos usos da terra, tais como residencial, recreativo, industrial/comercial e agrícola, mas irá mostrar a população 
distribuída uniformemente, embora a tendência seja que os habitantes se concentrem dentro da parte residencial (SLEETER, 2008).

O método mais amplamente aplicado para representar a informação demográfica é o coroplético (RICHARDS et al., 2010; BARROZO et al., 2016). Mapas temáticos poligonais que são elaborados com dados quantitativos e apresentam sua legenda ordenada em classes, conforme as regras próprias de utilização da variável visual valor por meio de tonalidades de cores, ou ainda, por uma sequência ordenada de cores que aumentam de intensidade conforme a sequência de valores apresentados nas classes estabelecidas (ARCHELA e THÉRY, 2008).

No entanto, polígonos em um mapa coroplético podem dar a noção errônea de distribuição homogênea sobre a área em situações em que a quantidade mapeada varia de acordo com sua distribuição espacial (DORLING, 1993; MENNIS, 2003; BARROZO et al., 2016). Em mapas de população, o mapa coroplético sugere distribuição uniforme de pessoas dentro de áreas administrativas de grande dimensão, entretanto a população é conhecida por ser distribuída de forma irregular dentro das unidades administrativas (BARROZO et al., 2016).

Dessa maneira, na busca por dados mais precisos e que melhor reflitam a realidade, os métodos de interpolação zonal têm sido largamente utilizados (SILVA, 2009). Tais métodos de interpolação de área são projetados principalmente para o problema da transformação zonal, que envolve a transferência de dados a partir de um conjunto de unidades espaciais para outro. Esta abordagem utiliza dados populacionais do censo como entrada e aplica técnicas de interpolação ou desagregação para obter superfícies ou áreas com população refinada (WANG et al., 2005; Silva, 2009).

Esses métodos são divididos em decorrência do uso ou não de dados auxiliares. Dentre os métodos que utilizam dados auxiliares destaca-se o método dasimétrico (SILVA, 2009).A metodologia dasimétrica permite a produção de mapas de distribuição visualmente mais comunicativos e precisos (LANGFORD, 2005) com estimativas populacionais mais detalhadas 
(MROZINSKI e CROMLEY, 1999; GREGORY, 2002; MENNIS e HULTGREEN, 2006).

Este método pode ser considerado uma sofisticação de um mapa coroplético, que utiliza dados auxiliares para refinar a representação da distribuição espacial da variável analisada (LANGFORD et al, 1991; EICKER e BREWER, 2001; MENNIS e HULTGREN, 2006), em oposição à premissa simplificadora usual, segundo a qual a variável se distribui uniformemente pelo espaço delimitado (UMBELINO, 2012).

O mapeamento dasimétrico é um tipo específico de interpolação zonal, baseada em uma técnica cartográfica, para desagregar variáveis demográficas como população total e densidade populacional (FRANÇA et al., 2014). Pode ser definido ainda como um tipo de mapa para exibição e visualização de dados estatísticos de uma área por meio do particionamento do espaço em zonas, que reflitam com maior fidelidade as variações das características da área em estudo (MENNIS e HULTGREN, 2006).

Este método surgiu na década de 20, sendo implementado pela primeira vez pelo cartógrafo Semenov-Tian-Shansky, em 1928, com o intuito de realizar a distribuição populacional da Rússia utilizando uma área de influência a partir do traçado de áreas edificadas (BIELECKA, 2005). Desde sua primeira utilização diferentes modos de aplicação têm sido desenvolvidos (LANGFORD e UNWIN, 1994; HOLLOWAY, 1996; MENNIS, 2002; AMARAL et al., 2005; NERY et al., 2007; FREIRE e GOMES, 2010; UMBELINO et al., 2012; BIAN e WILMOT, 2015; FRANÇA et al., 2014; BARROZO et al., 2016; ZAMBRANO, 2015).

Com a variedade de diversificações do método dasimétrico várias pesquisas (EICHER e BREWER, 2001; MENNIS e HULTGREEN 2006; LANGFORD et al., 2008; SILVA, 2009; BUENO e D'ANTONA, 2014) se destinaram ao estudo dos diferentes resultados obtidos por esses métodos e em compará-los. Cabe destacar a importância destes estudos para a composição dos projetos cartográficos, para além do detalhamento da informação, o método dasimétrico proporciona uma distribuição espacial e 
visual das informações mais adequada, melhorando a lingugem do mapa, uma vez que a eficiência das análises espaciais é dependente da comunicação cartográfica (SLUTER, 2008, SLOCUM et al. 2009; SLUTER et al. 2015 e).

Assim, este trabalho tem como objetivo comparar três diferentes abordagens do método dasimétrico para estimar a população urbana do Distrito Federal (Brasil).

\section{Materiais e Métodos}

O Distrito Federal - DF está localizado entre os paralelos de $15^{\circ} 30^{\prime}$ e $16^{\circ} 03^{\prime}$ de latitude sul e os meridianos de $47^{\circ} 25^{\prime}$ e $48^{\circ} 12^{\prime}$ de longitude oeste, no Planalto Central do Brasil. Ocupa área de $5.814 \mathrm{~km}^{2}$ e compreende a unidade da federação onde se situa a capital do país (Figura 1). A área efetivamente urbana, de acordo com o Plano Diretor de Ordenamento Territorial - PDOT (SEDUMA, 2009), corresponde a $1.179,37 \mathrm{~km}^{2} \mathrm{o}$ que corresponde a $20,33 \%$ do total do território. O DF possui uma divisão interna em Regiões Administrativas (RA), atualmente com trinta e uma RAs em sua configuração.

De acordo com a última estimativa do censo (IBGE, 2010) a população do DF é de 2.570.160 habitantes, com uma densidade populacional de 444,66 $\mathrm{hab} / \mathrm{km}^{2}$, sendo que deste montante cerca de $96 \%$ reside nas áreas urbanas (Figura 1). A população total estimada para o ano de 2015 é de 2.914 .830 habitantes, o que corresponde a um aumento de 13\% em cinco anos (IBGE, 2014). A previsão é que a população terá um crescimento para algo em torno de 3,5 milhões em 2025 e 3,7 em 2030 (IBGE, 2013). 
Figura 1- Localização da área urbana do Distrito Federal e suas Regiões Administrativas

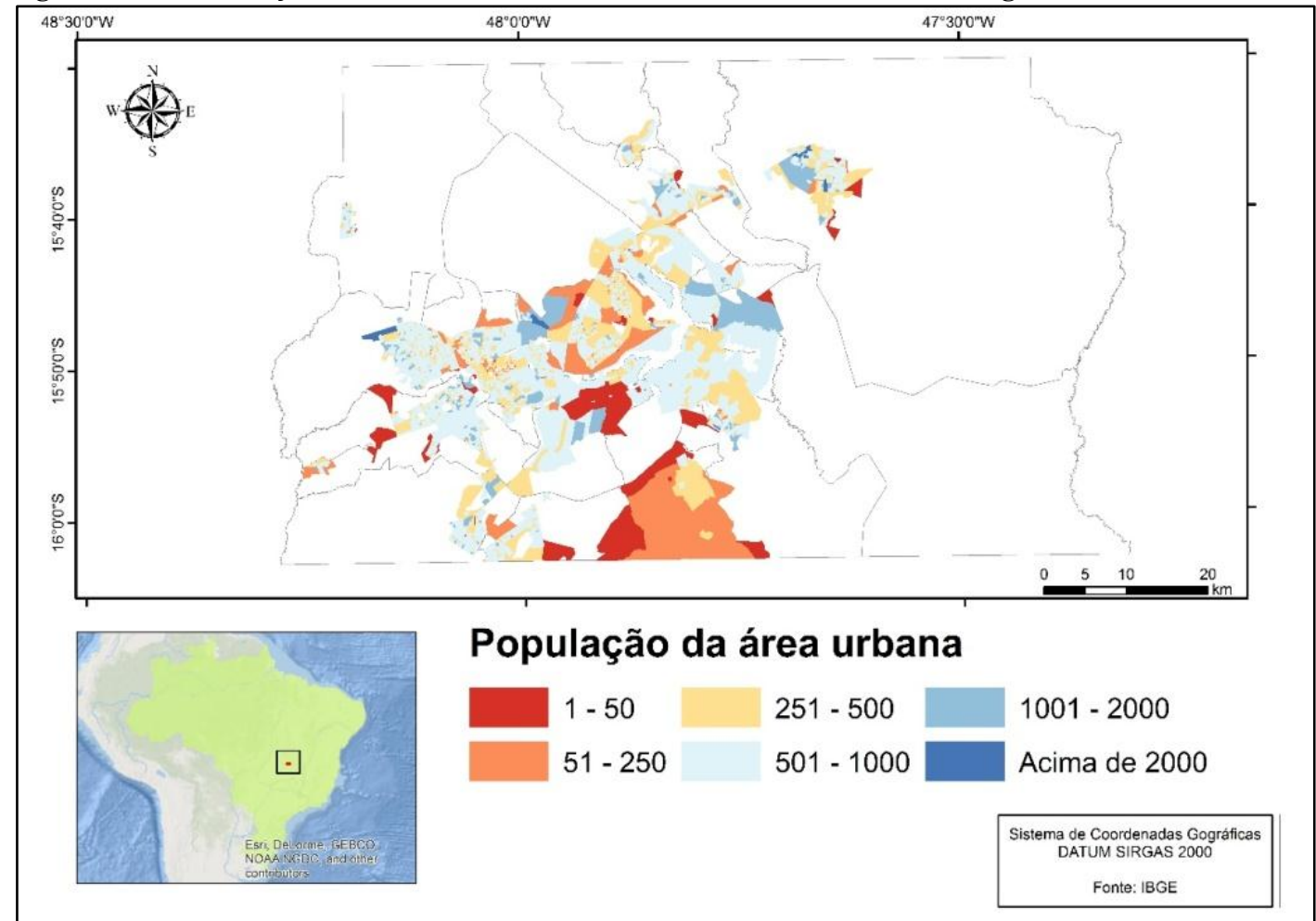

Fonte: Elaborada pelos autores.

O fluxograma da Figura 2 apresenta os métodos e procedimentos utilizados para a geração dos mapeamentos dasimétricos de acordo com três diferentes abordagens. Todo o procedimento foi realizado em base vetorial. De acordo com Eicher e Brewer (2001) e França et al. (2014) os métodos apresentaram erros menores quando desenvolvidos usando uma estrutura vetorial, embora a diferença não fosse significativa. 
Figura 2 - Fluxograma com procedimento para a geração dos modelos dasimétricos para a área urbana do DF.

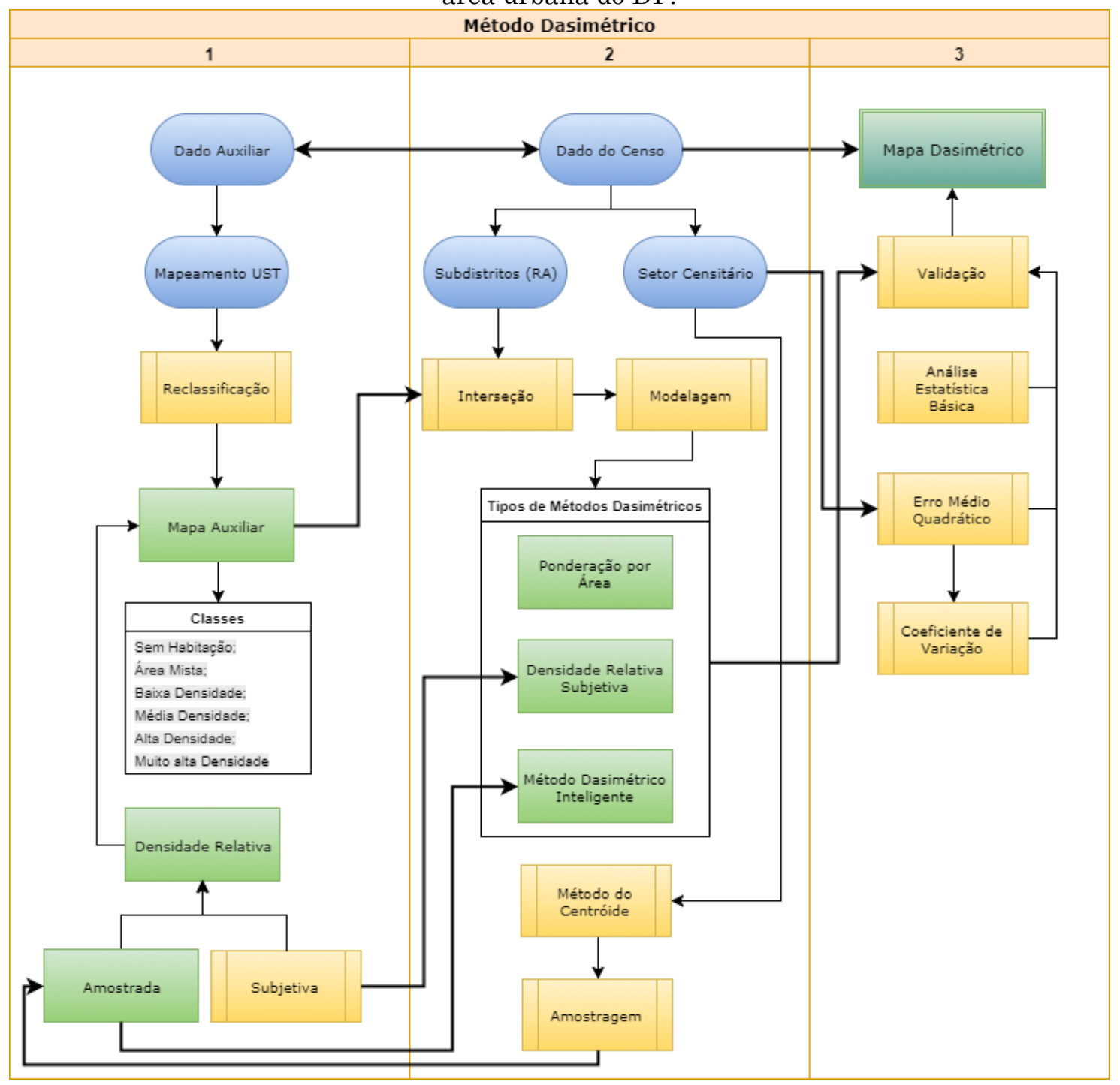

Fonte: Elaborada pelos autores.

A primeira abordagem se refere ao particionamento da população urbana do DF levando em consideração a ponderação por área (MD1). O segundo método é uma variação do método de três classes, o qual usa como informação auxiliar um mapa com três categorias (urbano, agrícola e floresta) e considera a variável densidade para o cálculo, sendo essa baseada em uma porcentagem definida de forma subjetiva dependendo do conhecimento empírico sobre a área (EICHER e BREWER, 2001). Para este estudo adotouse o uso de seis classes para o mapa auxiliar e para cada classe foi definido subjetivamente um valor de densidade relativa (MD2). O terceiro método foi 
baseado no mapeamento dasimétrico inteligente (MENNIS e HULTGREEN, 2006), o qual define a densidade relativa por meio de amostragem (MD3).

A base territorial do Censo (IBGE, 2011) foi criada buscando integrar a representação espacial das áreas urbana e rural do Território Nacional em um ambiente de banco de dados geoespaciais. Assim, os dados coletados serviram para a construção de cadastros territoriais e mapas digitais referentes aos municípios, às localidades e aos setores censitários (IBGE, 2011). Para a desagregação da população foi realizado o recorte para as áreas urbanas e posteriormente utilizado as informações disponíveis por subdistrito e a base poligonal dos setores censitários, para possibilitar a avaliação dos dados gerados.

Os subdistritos são unidades administrativas municipais, normalmente estabelecidas nas grandes cidades, criadas por meio de leis ordinárias das Câmaras Municipais e sancionadas pelo prefeito (IBGE, 2011). A divisão por subdistritos no caso do Distrito Federal compreende as Regiões Administrativas (RA), no ano de 2010 considerou-se 19 subdistritos. O setor censitário é a unidade territorial de controle cadastral da coleta, constituída por áreas contíguas, respeitando-se os limites da divisão políticoadministrativa, do quadro urbano e rural legal e de outras estruturas territoriais de interesse, além dos parâmetros de dimensão mais adequados à operação de coleta (IBGE, 2011).

O mapa de uso e ocupação da terra utilizado para gerar os mapas dasimétricos baseou-se no conceito de morfologia urbana. Trata-se do mapeamento da área urbana do DF segundo seus tipos de estruturas urbanas (UST, Figura 3A). O mapa possui uma escala de 1: 10.000 e foi realizado com ortofotos com 1 metro de resolução (CASTRO, 2017). O diferencial desse mapeamento, além do nível de detalhamento da escala, é que ele divide o espaço urbano considerando regiões homogêneas espacialmente em termos da relação entre os tipos de cobertura, tipo de uso, características físicas e funcionalidade (HETCH et al., 2013) e dessa forma possibilita uma divisão mais precisa do território. 
As classes do mapa UST foram agregadas de acordo com o adensamento urbano e o padrão construtivo nessas áreas, resultando em um mapa auxiliar (Figura 3B) composto por seis classes: Não habitado; áreas mistas; baixa aglomeração; média aglomeração; alta aglomeração e; muito alta aglomeração. As áreas consideradas mistas se referem a classes que se constituem de áreas comerciais e/ou industriais, mas que também possuem residências.

A classe "Não habitado" corresponde as áreas verdes intraurbanas, unidades de conservação, áreas degradas, áreas destinadas a plantio e pastagem e espaços abertos em geral que não são efetivamente ocupados, além das áreas urbanas onde é sabido que não se tem a função residencial como áreas exclusivas para comércio e indústria, instituições e áreas públicas e espaços destinadas a construção civil.

Para cada classe do mapa auxiliar foi atribuída uma densidade populacional $\left(\mathrm{hab} / \mathrm{m}^{2}\right)$ relativa $(\mathrm{DR})$ de acordo com cada método. Para o primeiro MD não se assumiu nenhuma densidade e as áreas da classe "Não habitado" foram excluídas do cálculo. Para o MD2 foi utilizada uma densidade subjetiva de acordo com o prévio conhecimento da área e para o MD3 foi realizada uma amostragem baseada no método do centroide (MARTIN, 1989; MENNIS e HULTGREN, 2006), o qual utiliza as zonas de origem cujos centroides se encontram no interior de uma dada classe. Para os métodos MD2 e MD3 a classe "Não habitado" teve a densidade relativa com valor igual à zero.

Mennis e Hultgreen (2006) realizaram uma comparação entre os diferentes tipos de amostragem (polígono totalmente inserido, polígono parcialmente inserido e método do centroide) e de variações destes, constataram que os diferentes métodos que utilizam também áreas previamente definidas (inabitada, por exemplo) apresentaram os melhores resultados e também resultados muito próximos. Dessa forma, método do centroide foi escolhido em detrimento dos outros, pois apresentou uma amostragem mais representativa e por ser o mais facilmente processado. 
Figura 3 - A) Mapa de tipos de estruturas urbanas do DF e; B) Mapa auxiliar das áreas urbanas do Distrito Federal.

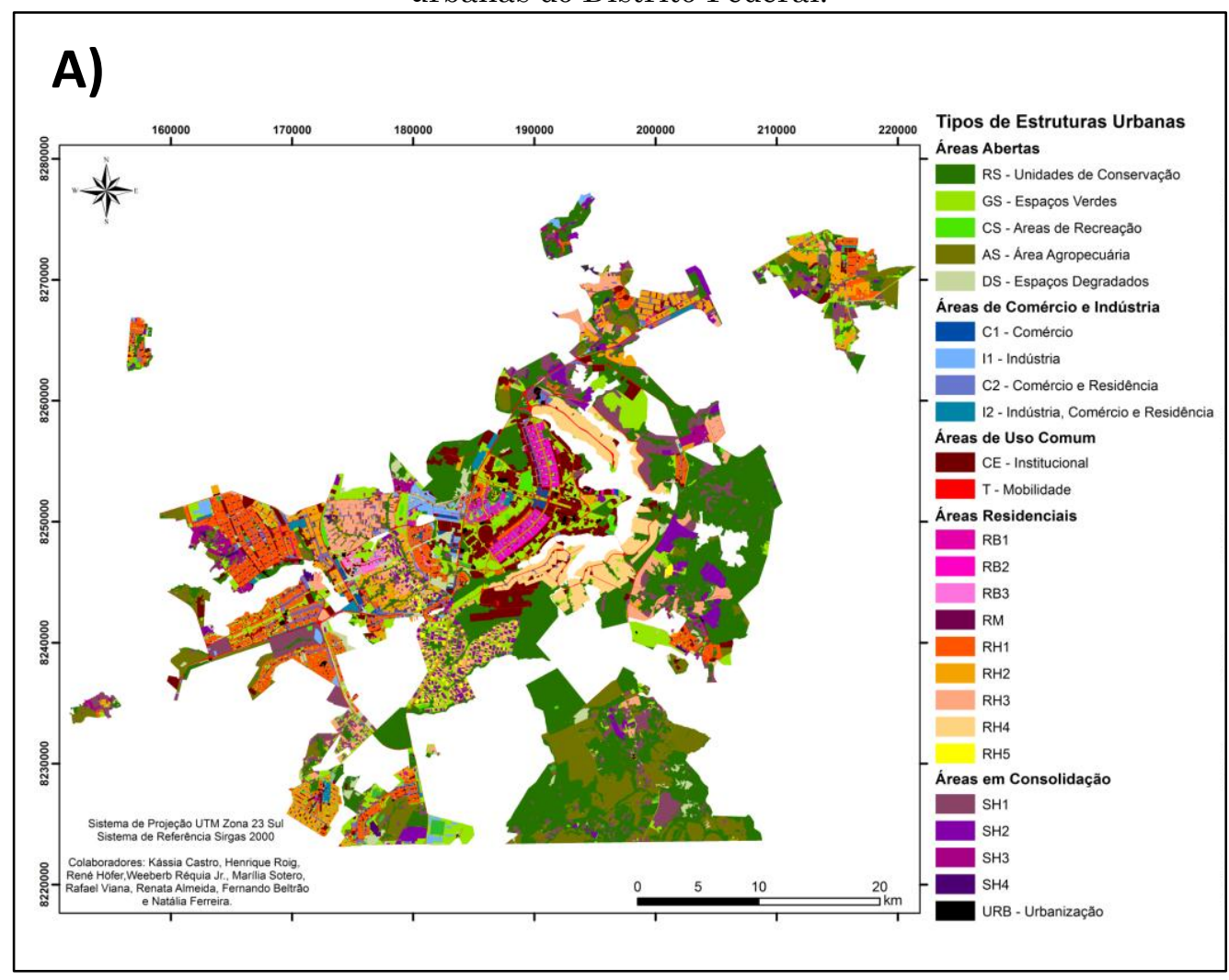

\section{B)}

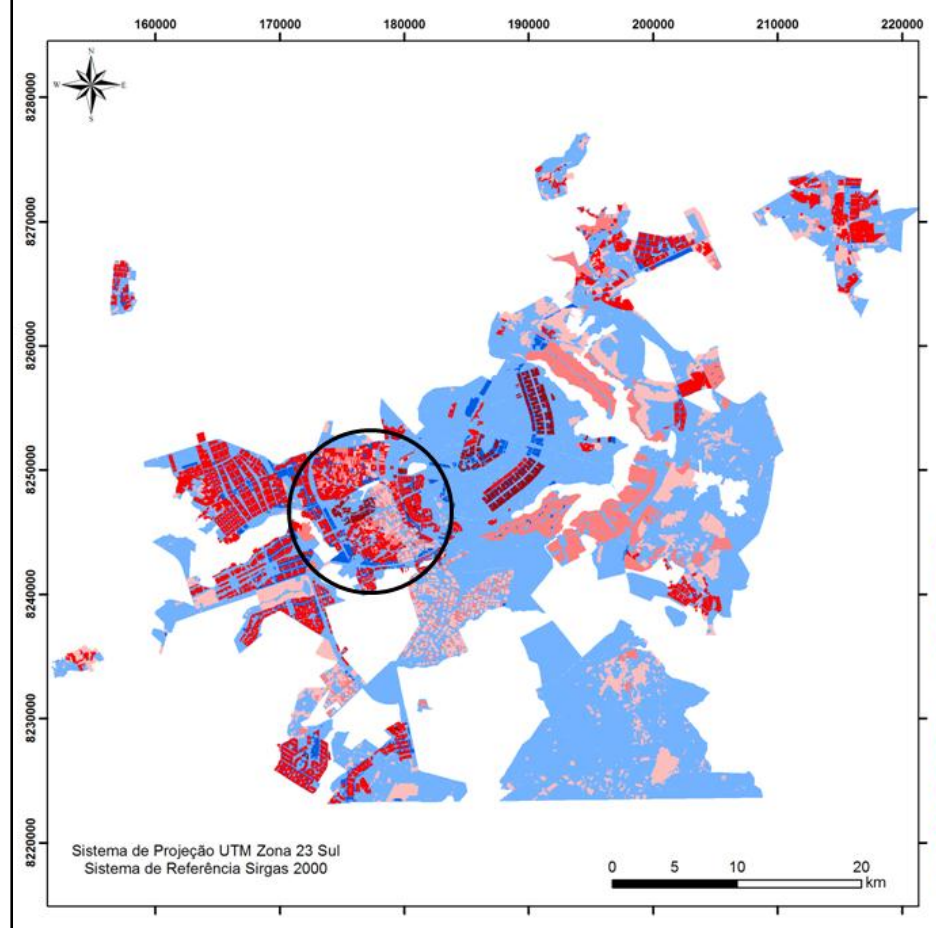

Legenda

Mapa Auxiliar

Não Habitado

Área Mista

Baixa Densidade

Média Densidade

Alta Densidade

Muito Alta Densidade

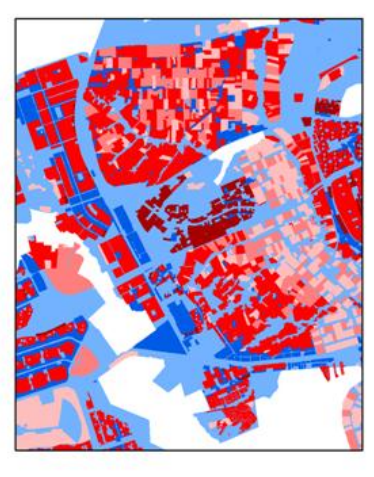

Fonte: Elaborada pelos autores.

Rev. Bras. Cartogr., vol. 71, n. 1, janeiro/março, 2019. pp. 207 - 232. 
Para esta amostragem baseou-se nos dados disponíveis por setor censitário. Após as amostras serem definidas para cada classe do mapeamento auxiliar, a densidade populacional relativa foi definida pela equação 1. Os valores de densidade relativa para cada classe são apresentados na tabela 1.

$$
\mathrm{D}_{\mathrm{c}}=\frac{\sum_{\mathrm{o=1}}^{\mathrm{j}} \mathrm{P}_{\mathrm{o}}}{\sum_{\mathrm{o}=1}^{\mathrm{j}} \mathrm{A}_{\mathrm{o}}}
$$

Onde:

$D_{c}=$ Fração de densidade correspondente as classes do mapa auxiliar;

$P_{o}=$ população conhecida na zona de origem $o$;

$A_{o}=$ área total na zona de origem $o$.

Tabela 1 - Valores de densidade relativa por classe para as diferentes formas de análise.

\begin{tabular}{l|c|c|c}
\hline \multicolumn{1}{c|}{ Classe } & DR subjetiva & \multicolumn{2}{c}{$\begin{array}{c}\text { DR amostrada/ quantidade de } \\
\text { polígonos }\end{array}$} \\
\hline Não habitado & 0 & 0 & - \\
\hline Área mista & 5 & 6.43 & 334 \\
\hline Baixa aglomeração & 5 & 0.23 & 115 \\
\hline Média aglomeração & 15 & 0.44 & 186 \\
\hline Alta aglomeração & 30 & 28.35 & 2196 \\
\hline Muito alta aglomeração & 50 & 12.04 & 602 \\
\hline
\end{tabular}

Fonte: Elaborada pelos autores.

Depois de definidas as densidades relativas para os mapas MD2 e MD3 realizou-se os procedimentos de intercessão do vetor de setores censitários com o mapa auxiliar, para a geração das classes de destino. As classes dos setores censitários foram previamente editadas para conter os dados populacionais referentes ao nível de subdistrito, unidades administrativas municipais, normalmente estabelecidas nas grandes cidades, criadas através de leis ordinárias do município (IBGE, 2011), no caso do DF trata-se das regiões administrativas vigentes até a data do censo. Após esse procedimento para cada um dos métodos (MD1, MD2 e MD3) o valor da população para cada 
área de destino foi estimado. Para o método MD1 foi realizada a seguinte equação (2):

$$
P_{d}=\frac{P_{o} A_{o n d}}{\sum_{d=1}^{n} A_{d}}
$$

Onde:

$P_{d}=$ população estimada para a zona de destino $d$;

$P_{o}=$ população conhecida na zona de origem $o$;

$A_{o n d}=$ interseção entre a área zona de origem $o$ e a área zona de destino $d$;

$A_{d}=$ área da zona de destino $d$;

$n=$ número de zonas de destino.

Para os mapas MD2 e MD3 a densidade relativa, subjetiva e amostrada respectivamente, foi inserida no cálculo conforme a equação 3.

$$
P_{d}=\frac{P_{o} A_{d} D_{c}}{\sum_{d \in o}\left(A_{d} D_{c}\right)}
$$

Onde:

$D_{c}=$ Fração de densidade correspondente as classes do mapa auxiliar.

Os mapas dasimétricos foram criados a partir dos dados agregados em uma unidade espacial maior (subdistritos) e comparados com a realidade conhecida na unidade menor (setor censitário). A avaliação de cada técnica foi realizada por meio de estatística básica, erro médio quadrático (RMSE) e do coeficiente de variação, equações 4 e 5 , respectivamente(EICHER e BREWER, 2001; MENNIS e HULTGREEN 2006; LANGFORD et al., 2008; SILVA, 2009; BUENO e D'ANTONA, 2014).

$$
R M S E_{o}=\sqrt{\frac{\sum(P s-P s)^{2}}{n}}
$$


Onde:

$R M S E_{o}=$ Erro médio quadrático na zona de origem;

$P s=$ População na zona de origem s;

$P s=$ População estimada na zona de origem s;

$n=$ Número de subseções contidas em $o$.

$$
C V_{O}=\frac{R M S E_{O}}{P_{O}}
$$

Onde:

$C V_{O}$ = Coeficiente de variação na zona de origem;

$P_{o}=$ população conhecida na zona de origem $o$.

Procedeu-se a análise em dois níveis e assumiu-se a hipótese que o método com melhor desempenho no maior nível de agregação também seria melhor no nível mais detalhado (WEBER, 2010; FRANÇA et al., 2014). Assim, utilizou-se o método que apresentou o melhor desempenho para a redistribuição da população também para o nível de informação do setor censitário.

\section{Resultados e Discussões}

Foram gerados três mapas dasimétricos utilizando-se as diferentes abordagens (MD1, MD2 e MD3). A figura 4 apresenta uma comparação entre a distribuição populacional do censo por setores censitários e por meio dos três métodos gerados.

Os três modelos estimaram o valor de população como sendo zero para a classe "Não habitado" conforme o esperado, de acordo com as metodologias propostas, essas áreas foram excluídas do mapa (Figura 4), para melhor visualização de onde de fato se concentra a população. Observando-se a figura 4 é possível perceber que todos os modelos apresentam diferenças entre si, entretanto, visualmente nota-se que os modelos MD1 e MD2 apresentaram maior número de áreas com valores absolutos de população superiores a 1000 habitantes e em contrapartida o modelo MD3 apresentou maior número de 
áreas com valores mais baixos de população (até 50 habitantes) que os outros dois modelos.

Figura 4 -A) População por setor censitário; B) População redistribuída pelo método MD1; C) População redistribuída pelo método MD2; D) População redistribuída pelo método MD3.

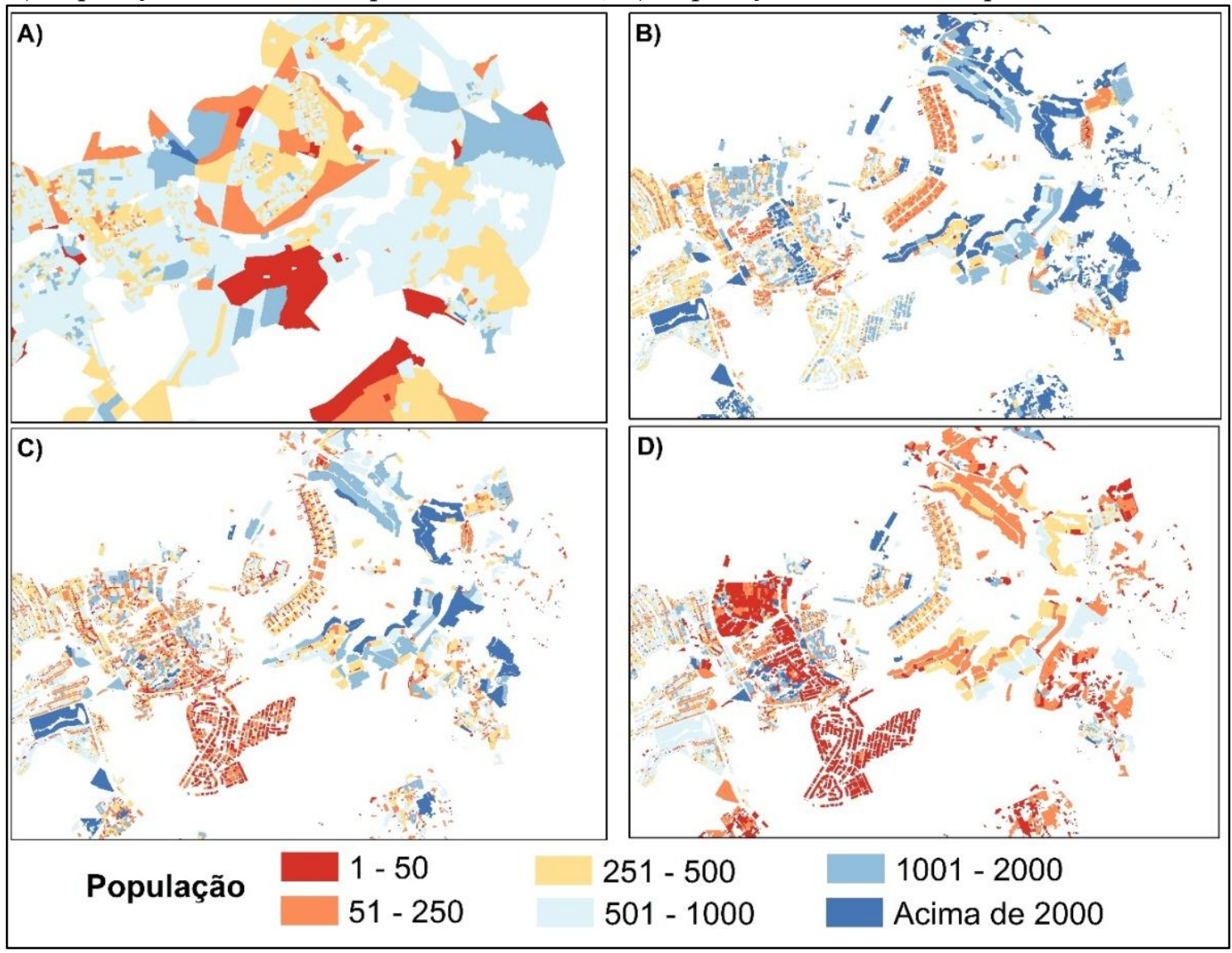

Fonte: Elaborada pelos autores.

De acordo com França et al. (2014), o resultado da abordagem dasimétrica é mais exato que o dado original agregado, entretanto, é necessário que se avalie os erros e incertezas dessa estimativa. Assim, todos os modelos foram avaliados buscando o entendimento das diferenças e capacidades de cada abordagem e visando não apenas apontar qual modelo mais eficiente, mas também identificar as inconsistências de cada um. A tabela 2 apresenta a estatística básica para cada um dos métodos e também dos dados do setor censitário como referência. 
Tabela 2 - Estatística básica gerada para os mapas dasimétricos gerados e para a população dos setores censitários para efeito de comparação.

\begin{tabular}{c|c|c|c|c}
\hline Estatística básica & $\begin{array}{c}\text { Setor } \\
\text { censitário }\end{array}$ & MD1 & MD2 & MD3 \\
\hline Média & 593,03 & 142,03 & 142,03 & 142,03 \\
\hline Erro padrão & 3,84 & 4,35 & 2,54 & 2,54 \\
\hline Desvio padrão & 248,19 & 573,75 & 335,81 & 335,70 \\
\hline Variância da amostra & 61599,75 & 329194,36 & 112768,37 & 112695,50 \\
\hline Curtose & 5,03 & 1746,65 & 183,54 & 54,78 \\
\hline Assimetria & 0,63 & 31,42 & 7,92 & 4,99 \\
\hline Intervalo & 3067,00 & 41023,25 & 13496,60 & 8497,68 \\
\hline Mínimo & 0,00 & 0,00 & 0,00 & 0,00 \\
\hline Máximo & 3067,00 & 41023,25 & 13496,60 & 8497,68 \\
\hline Soma & 2473527,00 & 2473527,0 & 2473527,00 & 2473527,00 \\
\hline Contagem & 4171,00 & 17415,00 & 17415,00 & 17415,00 \\
\hline
\end{tabular}

Fonte: Elaborada pelos autores.

O modelo MD1 apresentou os maiores valores para desvio padrão, variância, assimetria e curtose. Os modelos MD2 e MD3 apresentaram valores de desvio padrão e variância muito próximos, porém os valores de curtose e assimetria para MD3 foram significativamente menores.

O valor mínimo para todos os modelos foi igual a zero, correspondente principalmente as áreas referentes a classe "Não habitado" e a áreas sem população no dado original. Já o valor máximo mais alto foi estimado pelo MD1, cerca de treze vezes o valor máximo recenseado, o modelo MD2 também superestimou esse valor em cerca de quatro vezes e o MD3 mais que duas vezes.

Para melhor entendimento dos dados gerou-se gráficos de dispersão e o valor do $\mathrm{R}^{2}$ (coeficiente de determinação) para cada um dos modelos (Figura 5). O modelo MD1 apresentou a maior dispersão dos dados em torno da linha de tendência central e apresentou o menor $R^{2}$ igual a 0,009. Os modelos MD2 e MD3 apresentaram respectivamente coeficientes de 0,138 e 0,243. Todos os $\mathrm{R}^{2}$ apresentados são baixos, e, portanto, não explicam os valores observados. 
Figura 5 - Gráfico de dispersão dos dados dos setores censitários: A) dos dados gerados pelo MD1; B) dos dados gerados pelo MD2; C) dos dados gerados pelo MD3.
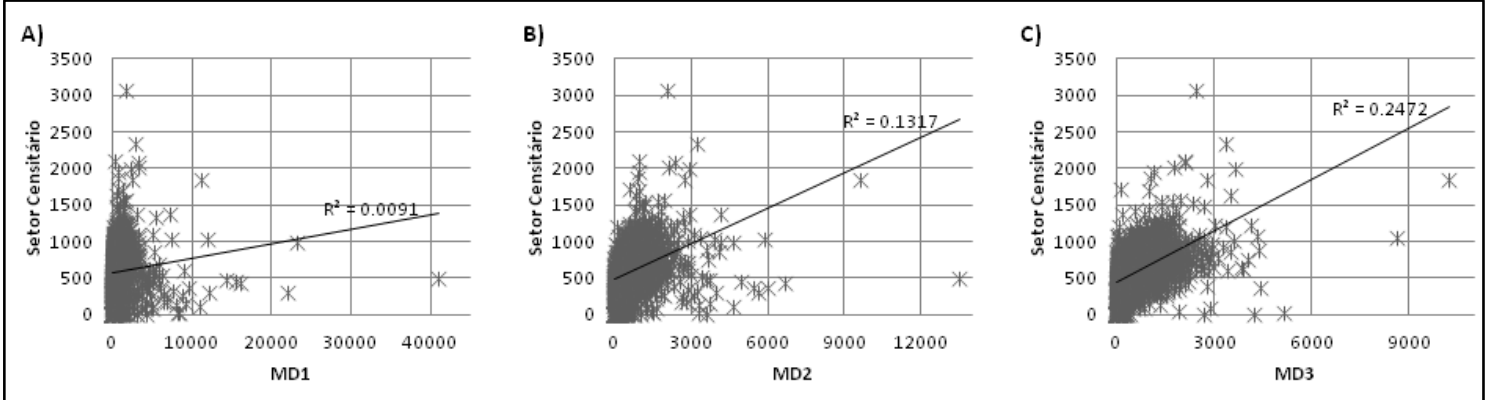

Fonte: Elaborada pelos autores.

Além da estatística básica e do coeficiente de determinação foram calculados também o erro médio quadrático (RMSE) e o coeficiente de variação. O MD1 apresentou os piores resultados com RMSE de 590,12 e coeficiente de variação de 0,00024. O modelo MD2 apresentou um RMSE igual a 256,89 um pouco mais alto que o MD3 que teve RMSE de 224,63. Para o coeficiente de variação o MD2 e MD3 apresentaram valores muito próximos 0,00010 e 0,00009 respectivamente, não apresentando uma diferença estatística significativa.

Com o intuito de identificar as maiores fontes de erro de cada mapa dasimétrico foram gerados mapas de distribuição espacial do erro absoluto por setor censitário (Figura 6). Os valores negativos são referentes aos dados que foram superestimados e os valores positivos os dados subestimados. As áreas em vermelho ou azul mais escuro são as zonas onde ocorreram os maiores erros, conforme essas tonalidades ficam mais claras significa que os erros foram mais amenos. 
Figura 6 - Mapas de distribuição espacial do erro absoluto por setor censitário em termos de desvio padrão para os modelos: A) MD1; B) MD2 e; C) MD3.

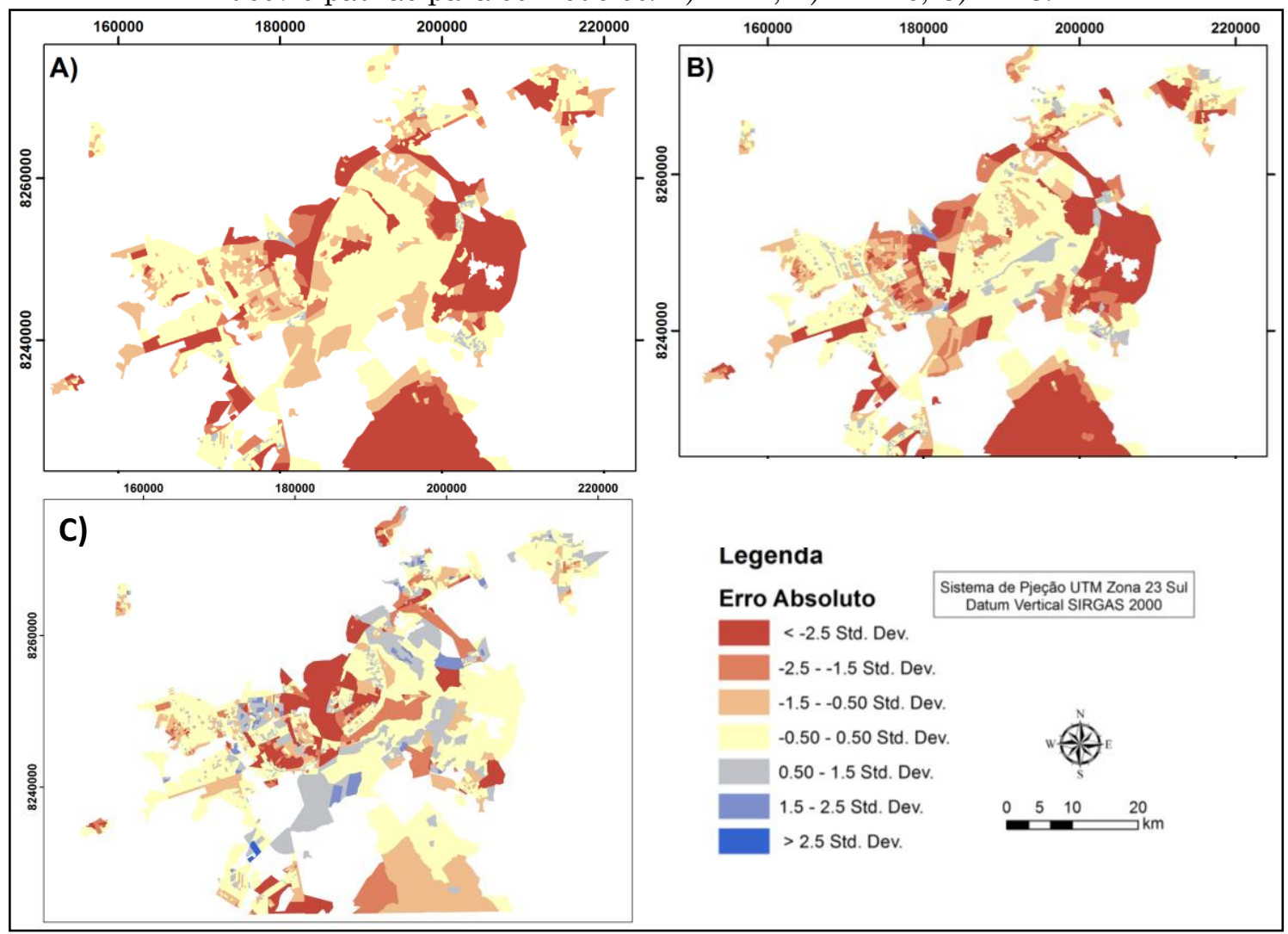

Fonte: Elaborada pelos autores.

De um modo geral o MD1 e MD2 superestimaram mais os valores absolutos de população, enquanto que o MD3 teve maior subestimativa. $\mathrm{O}$ MD3 apresenta áreas acima de 2,5 desvios padrão tanto positiva quanto negativamente. O que se observa (Figura 6) é que MD1 e MD2 tiveram maiores erros de estimativa nos setores censitários com maior área, o que os torna piores para a construção de representações visuais. Se compararmos esse resultado com o dado populacional do censo (setor censitário, Figura 1) pode-se aferir que na maior parte dos casos superestimados os maiores erros dos modelos MD1 e MD2 ocorreram em áreas com menor população (até 374 pessoas), já para o modelo MD3 o mesmo não se verifica.

De acordo com todos os dados gráficos e estatísticos apresentados nessa pesquisa, com exceção do coeficiente de variação, que não apresentou diferença significativa entre o MD2 e MD3, pode-se afirmar que, considerando o conjunto de dados aplicados, a abordagem dasimétrica que se mostrou mais 
adequada para a aplicação da estimativa populacional para as áreas urbanas do DF foi o mapeamento dasimétrico inteligente com o método de amostragem por centroide, pois conseguiu representar todo o conjunto de dados populacional, apresentou uma variação da distribuição dos dados menor que a dos demais e representou melhor as áreas com menores populações.

Trabalhos anteriores (SILVA, 2009; MAN e CHANDRA, 2013; BUENO e D'ANTONA, 2014) também corroboram para os resultados obtidos por essa pesquisa, apontando que mapeamentos dasimétricos que utilizam a abordagem inteligente têm obtido os resultados mais satisfatórios. Mennis e Hultgreen (2006) apontaram que os métodos de amostragem que utilizam também valores pré-definidos apresentaram os melhores desempenhos em sua análise. E que os métodos com os maiores índices de desempenho foram os que utilizaram os métodos do polígono totalmente inserido e do centroide. Porém, deve-se ressaltar que o problema potencial com esse tipo de amostragem é que é vulnerável a valores extremos, gerando amostras incorretas (MENNIS e HULTGREEN, 2006).

Silva (2009) para realizar a desagregação da população da região do Vale do Tejo em Portugal utilizou os métodos binário e MDI com dados auxiliares em diferentes escalas, para testar a diferença entre os tipos de metodologia e a influência do detalhamento da informação nos resultados. De acordo com ele, a técnica que utiliza o dado auxiliar mais detalhado juntamente com a técnica MDI apresentou os melhores resultados.

Além disso, os resultados menos satisfatórios foram verificados no modelo com o mapa auxiliar com escala de menor detalhe, mesmo com a utilização da abordagem MDI. Entretanto, para Bueno e D'Antona (2014) não existe um método de desagregação melhor que o outro, pois isso depende do objetivo da análise, da disponibilidade de informações para auxiliar a distribuição da população e das características de ocupação da área de estudo.

Dessa forma, após a avaliação de desempenho dos métodos aplicou-se a abordagem MD3 para o universo de informação em nível de setor censitário (Figura 7), acreditando na premissa que se para um nível mais amplo este foi 
o melhor estimador, para um nível mais detalhado também será a técnica que melhor se aplica. Como é possível perceber, mesmo utilizando-se a mesma abordagem, existe diferença entre os resultados, isso porque a informação do modelo da figura 7B é muito mais detalhada do que a utilizada pelo MD3 (Figura 7A).

Figura 7 - A) Modelo dasimétrico MD3 utilizando nível de informação de subdistrito; B) Modelo dasimétrico gerado com a mesma abordagem MD3, porém utilizando nível de informação de setor censitário.

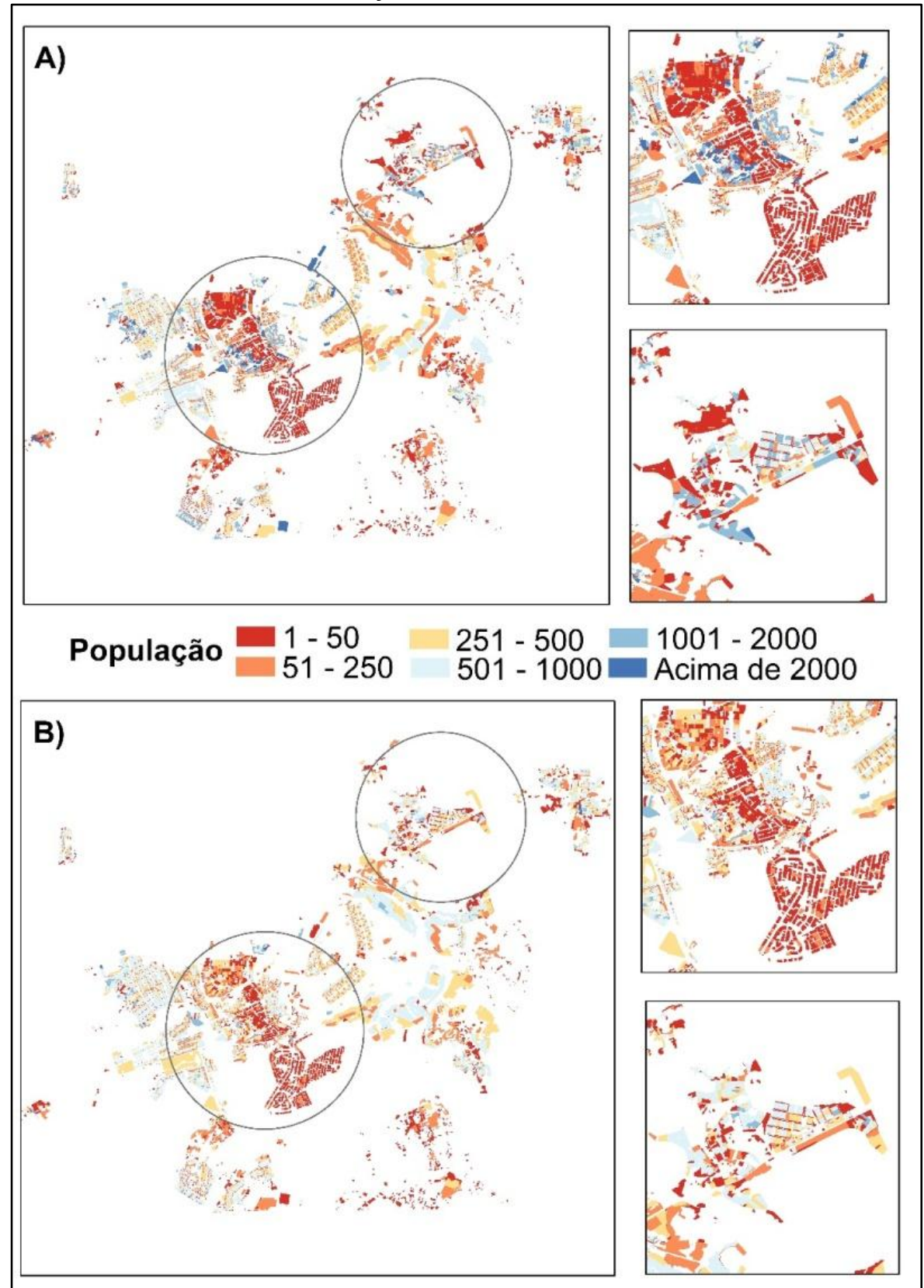

Fonte: Elaborada pelos autores. 
Assim, afirma-se que o dado gerado com a informação do setor censitário e a abordagem MD3 é o mais preciso, considerando que este método apresentou melhores resultados e que a informação no nível censitário possui uma agregação menor e por isso é mais exato.

\section{Conclusão}

Concluiu-se que a desagregação da população da área urbana do Distrito Federal considerando três métodos distintos e com mapa auxiliar com informações dos tipos de estruturas urbanas do DF melhorou o nível de detalhamento da informação auxiliar e também melhorou os resultados estimados, o que pode tornar o mapeamento mais preciso e acurado.

O modelo que apresentou os melhores resultados gráficos e estatísticos foi o modelo dasimétrico inteligente (MDI) que utiliza informações acerca da densidade populacional no cálculo.

No entanto, para uma avaliação mais rigorosa e adequada, considerando não apenas a eficiência do interpolador, mas também da capacidade de comunicação de cada um dos modelos, seriam necessários testes de percepção visual, que poderão ser incorporados ao trabalho em oportunidades futuras.

Acredita-se que o método de desagregação dasimétrico apresenta um grande potencial, não só para uma análise mais precisa das variáveis obtidas pelo censo, mas também para gerar indicadores e índices a respeito da qualidade e bem-estar da população, estudos de vulnerabilidade socioambiental e outros. Trata-se de uma ferramenta bastante promissora por possibilitar a utilização dedados disponíveis com um detalhamento espacial mais preciso. 


\section{Agradecimentos}

À CAPES, pela bolsa de doutorado para o primeiro autor. Ao laboratório de Sensoriamento Remoto e Análise Espacial (LSRAE) do IG/UnB pela infraestrutura computacional e à ESRI e IMAGEM pela disponibilização dos softwares utilizados, por intermédio do contrato No 2011 MLK 8733.

\section{Referências}

AMARAL, S.; MONTEIRO, A. M. V.; CÂMARA, G.; QINTANILHA, J. A. Estimativa da distribuição espacial da população na Amazónia brasileira com uso de sensoriamento remoto orbital: contribuições das imagens CCD/CBERS no caso do município do Marabá-PA. Anais do XII Simpósio Brasileiro de Sensoriamento Remoto, Goiânia, 2005. pp. 765-773.

ARCHELA, R. S.; THÉRY, H. Orientação metodológica para construção e leitura de mapas temáticos. Confins, n. 3, 2008.pp. 1-21.

BARROZO, L. V.; PÉREZ-MACHADO, R. P.; SMALL, C.; CABRAL-MIRANDA, W. Changing spatial perception: dasymetric mapping to improve analysis of health outcomes in a megacity. Journal of Maps, vol. 12, n. 5, 2016, pp. 1242 1247.

BIAN, R.; WILMOT, C. G. A spatio-temporal population distribution method for emergency evacuation: a case study of New Orleans. Journal of the Transportation Research Board, vol. 2532, 2015. pp. 1-18.

BIELECKA, E. A dasymetric population density map of Poland. Actas da International Cartographic Conference, Corunha, 2005. 10p.

BUENO, M. C. D.; D’ANTONA, A. O. Avaliação de métodos de desagregação para geração de grades de população. Revista Espinhaço, vol. 3, n. 1, 2014. pp. 127-137.

CARDOSO, M. A.; OLINTO, M. T. A.; SICHIERI, R. Avanços metodológicos em estudos populacionais em alimentação e nutrição. Cad. Saúde Pública, vol. 26, n.11, 2010. pp. 2006-2007.

CASTRO, K. B. Segurança Hídrica Urbana: Morfologia Urbana e Indicadores de Serviços Ecossistêmicos: Estudo de caso do distrito federal, Brasil. Tese de doutoramento.Universidade de Brasília, Instituto de Geociências, Brasília. 2017. 207p. 
CRUZ, B. D de O.; NOCKO, L. M.; SILVA, A. C. da C.; GUIMARÃES, P. M. N. 2016. Oferta de mão de obra no Distrito Federal: determinantes da participação na força de trabalho e efeitos da recessão econômica. Companhia de Planejamento do Distrito Federal,Texto para Discussão TD, n. 16, 2016. 36p.

DORLING, D. Map Design for Census Mapping. Cartographic Journal, vol. 30, 1993. pp. 167-83.

EICHER, C., BREWER, C. Dasymetric mapping and areal interpolation: implementation and evaluation. Cartography and Geographic Information Science, vol.28, n.2, 2001. pp. 125-138.

FRANÇA, V. O.; STRAUCH, J. C. M.; AJARA, C. Método dasimétrico inteligente: uma aplicação na mesorregião metropolitana de Belém. Revista Brasileira de Cartografia, vol. 66, n. 6. 2014. pp. 1395-1411.

FREIRE, S. M. C. Modeling day time and night time population distribution in Portugal using geographic information system. Dissertação de Mestrado, Universidade do Kansas, Escola de graduação do Kansas. 2007.109p.

FREIRE, S.; GOMES, N. Aplicação de mapeamento dasimétrico inteligente na modelação da distribuição espácio-temporal da população na Área Metropolitana de Lisboa. Actas do XII Colóquio Ibérico de Geografia, 2010, Porto. 2p.

GODFRAY, H. C. J.; BEDDINGTON, J. R.; CRUTE, I. R.; HADDAD, L.; LAWRENCE, D.; MUIR, J. F.; PRETTY, J.; ROBINSON, S.; THOMAS, S. M.; TOULMIN, C. Food security: the challenge of feeding 9 billion people. Science, vol. 327, n. 5967, 2010. pp. 812-818,

GREGORY, I. N. The accuracy of areal interpolation techniques: standardizing 19th and 20th century census data to allow long-term comparisons. Computers, Environment and Urban Systems, vol. 26, 2002. pp. 293-314.

HECHT, R.; HEROLD, H.; MEINEL, G.; BUCHROITHNER, M. Automatic derivation of urban structure types from topographic maps by means of image analysis and machine learning. Proceedings of the 26th International Cartographic Conference, Dresden, 2013.18p.

HOLlOWAY, S. R.; SHUMACHER, J.; REDMOND, R. People and Place: dasymetric mapping using Arc/Info. Missoula: Wildlife Spatial Analysis Lab, University of Montana. 1996.

IBGE, Instituto Brasileiro de Geografia e Estatística. 2010. Site<http://censo2010.ibge.gov.br/pt/>acessado em setembro de 2014 . 
IBGE, Instituto Brasileiro de Geografia e Estatística. Base de informações do censo demográfico 2010: resultados do universo por setor censitário. Ministério do Planejamento, Orçamento e Gestão, Centro de Documentação e Disseminação de Informações, Rio de Janeiro, 2011. 201p.

IBGE, Instituto Brasileiro de Geografia e Estatística. Projeção da População do Brasil e Unidades da Federação por Sexo e Idade para o período 20002030. Ministério do Planejamento, Orçamento e Gestão, Diretoria de Pesquisas, Coordenação de População e Indicadores Sociais, Rio de Janeiro,2013. 21p.

IBGE, Instituto Brasileiro de Geografia e Estatística. Pesquisa Nacional por Amostra de Domicílios Contínua: Notas Metodológicas. Ministério do Planejamento, Orçamento e Gestão, Diretoria de Pesquisas Coordenação de Trabalho e Rendimento, Rio de Janeiro, v. 1, 2014. 47p.

LANGFORD, M. Dasymetric mapping of industry and commerce across Wales, UK, using geospatial integration of national mapping agency products. Congresson Surveying and Mapping, Las Vegas, 2005. 15p.

LANGFORD, M.; HIGGS, G.; RADCLIFFE, J.; WHITE, S. Urban population distribution models and service accessibility estimation. Computers, Environments and Urban Systems, vol. 32, 2008. pp. 66-80.

LANGFORD, M.; MAGUIRE, D. J.; UNWIN, D. J. The areal interpolation problem: Estimating population using remote sensing in a GIS framework. In: Handling geographic information: Methodology and potential applications. Masser, I., Blakemore, M. Londres: Longman. 1991. pp.55-77.

LANGFORD, M. e UNWIN, D.J. Generating and mapping population density surfaces within a geographical information system. CartographicJournal,vol.31, n.1, 1994.pp. 21-26.

MANN, D.; CHANDRA, O. B. C. Creating a gridded population data of India: a comparison and avaluation study of a dasymetric mapping methods. 14 ESRI India User Conference, Delhi, 2013.pp. 1-6.

MARTIN, D. Mapping population data from zone centroid locations. Transactions of the Institute of British Geographers, New Series, vol. 14, n. 1, 1989. pp. 90-97.

MENNIS, J. Using Geographic Information Systems to Create and Analyze Statistical Surfaces of Population and Risk for Environmental Justice Analysis. Social Science Quarterly, vol. 83, n. 1, 2002. pp. 281-297.

MENNIS, J. "Generating Surface Models of Population Using Dasymetric Mapping, "The Professional Geographer, vol. 55, n. 1, 2003. pp. 31-42. 
MENNIS, J., HULTGREN, T. Intelligent dasymetric mapping and its application to areal interpolation. Cartography and Geographic Information Science, vol.33, n.3, 2006. pp.179-194.

MROZINSKI, R. D. JR. and CROMLEY, R.G. Singly and doubly constrained methods of areal interpolation for vector-based GIS. Transactions in GIS, vol. 3,1999. pp. 285-301.

NÉRY, F.; MONTERROSO, P.; SANTOS, A.; MATOS, J. Interpolação zonal de estatísticas socio-económicas. Actas da V Conferência Nacional de Cartografia e Geodesia. Lisboa, 2007. pp. 89-100.

ONU, Organização das Nações Unidas. 2014.

Site<http://www.unric.org/pt/actualidade/31537-relatorio-da-onu-mostrapopulacao-mundial-cada-vez-mais-urbanizada-mais-de-metade-vive-emzonas-urbanizadas-ao-que-se-podem-juntar-25-mil-milhoes-em2050>acessado em agosto 2016.

ONU, Organização das Nações Unidas. 2015. Site< http://www.unric.org/pt/actualidade/31919-onu-projeta-que-populacaomundial-chegue-aos-85-mil-milhoes-em-2030>acessado em agosto 2016.

ONU, Organização das Nações Unidas. 2016. Site<http://www.un.org/en/development/desa/population/publications/pdf/urb anization/the_worlds_cities_in_2016_data_booklet.pdf $>$ acessadoemsetembro 2017.

RÉQUIA JR., E. J.; ROIG, H. L.; KOUTRAKIS, P. A novel land use approach for assessment of human health: The relationship between urban structure types and cardio respiratory disease risk. Environment International. vol. 85, 2015. pp. 334-342.

RICHARDS, T. B., BERKOWITZ, Z., THOMAS, C. C., FOSTER, S. L., GARDNER, A., KING, J. B.; ROYALTY, J. Choropleth map design for cancer incidence, part 1. Preventing Chronic Disease Public Health Research, Practice and Policy, vol. 7, n. 1, 2010. pp. 1-6,

SILVA, F. B. Modelação cartográfica e ordenamento do território: Um ensaio metodológico de cartografia dasimétrica aplicado a região oeste e Vale do Tejo. Dissertação de mestrado. Universidade do Porto, Faculdade de Letras, Porto. 2009. 177p.

SEDUMA, Secretaria de Estado de Desenvolvimento Urbano e Meio Ambiente. Plano Diretor de Ordenamento Territorial do Distrito Federal. 2009.

SEMENOV-TIAN-SHANSKY, B. Russia: Territory and population: a perspective on the 1926 census. Geographical Review, vol. 18, n. 4, 1928. pp. 616-640. 
SLEETER, R. A New Method for Mapping Population Distribution: U. S. Geological Survey Factsheet, 2008. pp. 2008-3010.

SLEETER, R. AND WOOD, N. Estimating daytime and nighttime population density for coastal 17 communities in Oregon. Urban and Regional Information Systems Association Annual 18 Conference Proceedings, Vancouver, 2006.pp. 1-15.

SLOCUM, A. T.; MCMASTER, R. B.; KESSLER, F. C.; HOWARD, H. H. Thematic Cartography and Geovisualization. 3nd ed. Prentice Hall, 2009. 624p.

SLUTER, C.R.. Uma abordagem sistêmica para o desenvolvimento de projeto cartográfico como parte do processo de comunicação. Portal de Cartografia, vol. 1, 2008. pp. 1-20.

SLUTER, C.R.; Brandalize, M. C. B. ;Ivánová, I. ; Elzakker, C. . Projeto cartográfico centrado no usuário dos mapas necessários à definição da estrutura do sistema viário de um plano diretor municipal. RBC. Revista Brasileira de Cartografia (Online), vol. 67/8, 2015. pp. 1557-1567.

UMBELINO, G. J. M.; BARBIERI, A. F.; DAVIS JR, C. A. Geotecnologias aplicadas a projeções domiciliares intraurbanas por quadras de Belo Horizonte 2010 a 2030. Anais do XVIII Encontro Nacional de Estudos Populacionais, Águas de Lindóia,2012. 20p.

WEBER, E. M. DasyTools: A user-friendly dasymetric mapping toolkit with automated accuracy assessments. Tese de doutoramento. University of Kansas, Department of Geography, Lawrence, 2010.105p.

WIEDMANN, T. O.; SCHANDL, H.; LENZEN, M.; MORAN, D.; SUH , S.; WEST, J.; KANEMOTO. K. The material footprint of nations. PNAS, vol. 112, n. 20, 2015. pp.6271-6276.

ZAMBRANO, G. J. D. Modelagem e representação espacial populacional no litoral de Santa Catarina a partir de tecidos urbanos identificados em imagens OLS. Dissertação de Mestrado. Universidade do Vale do Itajaí, Centro de Ciências da Terra e do Mar, Itajaí. 2015.54p. 